\title{
Barriers and Facilitators to Immediate Insertion of the Copper IUD after Second-Trimester Medical Abortion - Process Evaluation of A Randomized Controlled Trial
}

\section{Oluwaseyi Dolapo Somefun}

University of Cape Town School of Public Health and Family Medicine

Deborah Constant ( $\nabla$ deborah.constant@uct.ac.za )

Women's Health Research Unit, University of Cape Town https://orcid.org/0000-0002-7176-9963

Margit Endler

Karolinska Institutet

\section{Research}

Keywords: Second trimester medical abortion, copper IUD, Process Evaluation, South Africa,

Posted Date: February 4th, 2021

DOl: https://doi.org/10.21203/rs.3.rs-164796/v1

License: (c) (1) This work is licensed under a Creative Commons Attribution 4.0 International License.

Read Full License 


\section{Abstract}

\section{Introduction}

The availability of modern contraception including long-acting reversible contraceptives (LARC), is a fundamental component of post-abortion care. A randomized controlled trial (RCT) in South Africa compared immediate to interval insertion of the copper intrauterine device (IUD) after second trimester medical abortion (MA). Immediate insertion resulted in higher use at 6 weeks post-abortion, however expulsion rates were higher than interval insertion. This process evaluation sheds light on barriers and facilitators to the implementation of the study intervention.

\section{Methods}

We performed a process evaluation alongside the RCT in which we reviewed clinical records for fidelity to the RCT protocol and conducted in-depth interviews with 14 staff providing healthcare to RCT participants and 24 RCT participants. Research questions explored barriers and facilitators to implementation of immediate IUD insertion, contraceptive decision-making, and the potential impact of context and supplementary trial activities on trial findings. Interviews were recorded and transcribed, with translation into English if needed. We performed a triangulated thematic analysis at the level of the transcribed interview text.

\section{Results}

In the RCT, there were 8 crossovers from the immediate to the delayed arm: 5 had a clinical contraindication to the IUD post-MA and 3 changed their mind about the IUD. In deviation of the RCT protocol 10 women in the delayed arm were given the 3 monthly injectable instead of oral contraceptives. Doctors and nurses were generally in favour of immediate insertion and said it could be incorporated into standard care if women wanted this. This contrasted with the need for interventions by the research team to reinforce adherence by staff to the allocated intervention over the trial duration. For women, convenience, protection from pregnancy and privacy issues were paramount and they expressed preference for engagement with staff who knew their abortion history, and with whom they had an established connection.

Clinical trials registration: clinicaltrials.gov/ (ID NCT03505047), Pan African Trials Registry (www.pactr.org), ID PACTR201804003324963

\section{Conclusions}

Women and staff favour immediate IUD insertion after second trimester medical abortion, but service delivery may require structures that ensure continuity of care, communication that mitigates loss to follow up and training of staff to ensure competence.

\section{Contributions To The Literature}


- Providing immediate postabortion contraception improves reproductive health but efficient delivery is a challenge.

- In a randomized clinical trial we found that immediate compared to delayed insertion of copper IUD post-abortion at 17-20 weeks resulted in higher use. Our process evaluation identified contextual barriers and facilitators that may affect implementation of this policy in practice.

- Women and providers prefer immediate IUD insertion even though it not routine practice today. Additional staffing, task-shifting and continuous training are required for immediate insertion to be possible. Continuity of care, non-judgemental communication and convenient follow-up can improve uptake and adherence to follow-up among women.

\section{Introduction}

Comprehensive abortion care including postabortion contraception reduce recurring unintended pregnancies, increase birth spacing and improve maternal health [1]. Among South African women having a pregnancy over a five-year period, $66 \%$ reported the pregnancy was unintended [2]. While longacting reversible contraception methods (LARC) such as the IUD are most effective for planning and spacing pregnancies [3], use of the IUD in South Africa is low, at 3\% [2].

Barriers to effective contraceptive uptake and use persist in South Africa and include poor provider attitudes, lack of training in LARC insertion, long waiting hours, little or no contraceptive counselling, and high rates of discontinuation [4]. Healthcare centres mandated to provide postpartum and postabortion contraception are routinely overburdened resulting in short staff-to-patient interactions and quick-fix contraceptive provision.

Between 2018 and 2019, we conducted a randomized controlled trial (RCT) in South Africa comparing immediate and delayed insertion of the Copper IUD after medical abortion at 17-20 gestational weeks [BMJSRH: In press]. We found significantly higher rates of IUD use at 6 weeks, 3 and 6 months after immediate insertion, in large part because women failed to come for delayed insertion. Expulsion rates after immediate insertion were also higher. During the study we saw deviations from protocol by clinical staff as well as unexpected decision-making by women. These findings warranted exploration to address the feasibility of implementing immediate IUD insertion in this setting and outside of the study context.

\section{Rct Procedures}

We assessed the safety, effectiveness and acceptability of immediate compared to delayed insertion of the copper-IUD after medical abortion (MA) at 17-20 weeks GA in a randomized controlled trial (RCT) conducted in, South Africa between August 2018 and June 2019. In the study setting women requesting abortion in the second trimester are referred from their community health facilities (CHF) for a clinical assessment at a centralized hospital outpatient department prior to hospital admission. Women in the RCT immediate arm had an IUD inserted prior to discharge and within 24 hours of abortion completion. 
Those in the delayed arm were given one month's supply of oral contraceptive and referred to their local $\mathrm{CHF}$ for IUD insertion three weeks later. All women were planned for clinical follow-up with an ultrasound examination after 6 weeks. Fieldworkers performed follow-up phone interviews after 3-and 6 months. Primary outcomes for the RCT were 1) use of the original IUD, and 2) use of any IUD at the end of the 6week follow-up period.

Supplemental trial activities to support the safe and effective implementation of the study protocol included 1) pre-abortion group contraceptive counselling at the hospital for all women seeking abortion, 2) a booked 6-week consultation with the study clinician instead of a standard un-booked outpatient visit, 3 ) voice calls and text reminders to reschedule missed appointments and monthly reminders to complete the 6-week, 3- and 6-month follow-ups. R150 ( USD10) compensation was paid to participants in cash or as airtime on completion of each follow up appointment.

Supplemental activities for clinic nurses and hospital resident doctors, were 1) orientation and training in IUD insertion and 2) WhatsApp-mediated reminders to perform the insertion on the required day.

\section{Clinical trials registration}

clinicaltrials.gov/ (ID NCT03505047), Pan African Trials Registry (www.pactr.org), ID PACTR201804003324963

\section{Process Evaluation}

Process evaluations (PE) look beyond the effect size of an intervention and seek to clarify how it was achieved and the possible effects of contextual factors on study outcomes (5). We conducted this PE alongside the RCT. The aims were to report on 1) delivery of and fidelity to the RCT protocol, 2) barriers and facilitators to the implementation of immediate or delayed IUD uptake, 3) influences on women's contraceptive-decision making, and 4) the impact of trial procedures and context on trial outcomes.

\section{Method}

\section{Study design}

We reviewed study documents and clinical records to assess the delivery and fidelity of trial processes. We conducted in-depth interviews with healthcare staff and with selected women participating in the RCT. We developed semi-structured interview guides adapted to the role of clinical staff in study procedures, and the study arm to which women belonged. Interviews with resident doctors were done after they completed their rotation in the facility and clinic staff were interviewed at close-out of the RCT. Women were offered participation after their 3- and 6-month RCT follow-ups. To reduce observer bias interviews were conducted by staff who had not been involved in the RCT, however none were blinded to RCT outcomes as interview scripts differed accordingly. 


\section{Population And Sampling}

The RCT was implemented in a tertiary level teaching public hospital providing second trimester medical abortion, being the main referral centre for CHFs in that metro. Eligible women were $\geq 18$ years old, undergoing medical abortion between 17 and $20 \mathrm{GW}$, and opting and eligible for the copper IUD as postabortion contraception. For the PE, we selected participants to ensure that both RCT study arms were represented as well as the range of trial outcomes including use of the original IUD as planned, use of a replacement IUD, or non-use of an IUD. We included all resident doctors and nurses at the hospital involved in second trimester medical abortion care, as well as all primary healthcare nurses who had provided the IUD to $>2$ RCT participants $t$ in the PE.

\section{Data Collection}

A female medical doctor performed the telephone interviews with residents. Experienced qualitative research assistants, fluent in English and IsiXhosa, performed interviews in-person with hospital and clinic nurses and women participants. All interviewees gave written informed consent prior to being interviewed. Interviews were recorded, translated into English where needed and transcribed. Table 1 shows roles in the service for staff and randomization group and primary outcomes for women. Healthcare staff were not compensated for their time, women were given a ZAR180 ( 12 USD) food voucher to compensate for their time and transport costs. 
Table 1

Description of participants in process evaluation study describing barriers and facilitators to immediate insertion of the copper IUD after second-trimester medical abortion - a randomized controlled trial

\begin{tabular}{|c|c|c|}
\hline \multicolumn{3}{|l|}{ Staff } \\
\hline Facility & Designation & $\begin{array}{l}\text { Number of } \\
\text { respondents }\end{array}$ \\
\hline Hospital & Study doctor & 1 \\
\hline Hospital & Resident doctor & 6 \\
\hline Hospital & Senior registered nurse - ward manager & 1 \\
\hline Hospital & Enrolled nurse & 1 \\
\hline Hospital & Registered nurse & 1 \\
\hline $\begin{array}{l}\text { Community } \\
\text { healthcare } \\
\text { facility (CHF) }\end{array}$ & Doctor in charge of Family Planning & 1 \\
\hline $\mathrm{CHF}$ & Registered nurse; abortion care provider & 2 \\
\hline $\mathrm{CHF}$ & Registered nurse; abortion care manager & 1 \\
\hline \multicolumn{3}{|l|}{ Women } \\
\hline $\begin{array}{l}\text { Randomization } \\
\text { group }\end{array}$ & Uptake and Use of IUD & $\begin{array}{l}\text { Number of } \\
\text { respondents }\end{array}$ \\
\hline Immediate group & Got IUD as planned, IUD in situ at 6 weeks & 6 \\
\hline Immediate group & $\begin{array}{l}\text { Got } 1 \text { st IUD at } 6 \text { weeks (crossed-over to delayed group) or got } \\
\text { replacement IUD at } 6 \text { weeks (1st IUD expelled or malpositioned) }\end{array}$ & 6 \\
\hline Immediate group & IUD expulsed and not replaced & 1 \\
\hline Delayed group & Got IUD at $\mathrm{CHC}$ as planned & 3 \\
\hline Delayed group & Got 1 st IUD at 6 weeks study visit at hospital & 6 \\
\hline Delayed group & Not using an IUD & 2 \\
\hline
\end{tabular}

\section{Data Analysis And Ethics}

Two researchers (DC and ME) reviewed RCT case report forms and logs, and clinic records for information on delivery of the trial protocol. Items reviewed included numbers receiving the allocated intervention, protocol deviations, and relevant outcomes that affected trial outcomes such as abortion complications, time from abortion completion to IUD insertion, and reported difficulties with IUD insertion. 
Two researchers (OS and DC) analysed the text data extracted from interviews by thematic analysis. We performed several readings of the interview transcripts and coded and categorized the data using NVivo 8 qualitative data analysis software. Some categorizations were revised after discussion with ME.

\section{Results}

\section{Randomized controlled trial main outcomes}

The RCT intention to treat (ITT) analysis included 112 women, 55 and 57 in the immediate (I) and delayed (D) arms respectively. By ITT, $82 \%$ (I) and 21\% (D) of women received the IUD as planned in the study. At 6 weeks 56\% (I) and 19\% (D) were using the original IUD and 76\% (I) and 40\% (D) were using either the original, a replacement or a newly placed IUD. Two women has recurrent pregnancies within the 6 months trial follow-up period, both followed the delayed protocol. There were no serious adverse events.

\section{Process Evaluation Findings}

\section{Delivery of the trial protocol - Fidelity and dose}

Five women crossed over from the immediate to the delayed group after the abortion due to excessive bleeding, prolonged placental retention or suspected infection. Of these, 3 were provided with 1 months' supply of oral contraceptives, (OCs) as per study protocol, and 2 were given the 3-month injectable progestin contraceptive in deviation of protocol. There were 13 further protocol deviations: in the immediate group 2 changed their mind about the IUD after the abortion in favour of an injectable method, and 1 was given the injectable contraceptive in error. In the delayed group 10 were given the 3-month injectable contraceptive in place of OCs.

\section{In-depth Interviews}

We identified four main themes and twelve subsidiary categories to these themes.

1. Perspectives on the IUD and timing of insertion after medical abortion.

a. Preferences for immediate versus delayed insertion of the IUD

b. Barriers to immediate insertion of the IUD

c. Mechanisms to support immediate insertion of the IUD

2. Follow-up: Continuity of care and women's decisions at follow-up.

a. Continuity of care following abortion 
b. Women's decisions regarding the IUD during follow-up

3. Impact of study procedures on perceptions, behaviour and outcomes.

a. Group contraceptive counselling- effect on IUD uptake and women's experiences

b. Training

c. WhatsApp communication doctor's group

d. Communications between study staff and participants

e. Impact of trial on participant or provider behaviour

4. Contextual factors impacting on trial outcomes.

a. Staff shortages infrastructure and resource constraints

b. Women's perceptions of primary healthcare services

\section{Theme 1: Perspectives on timing for IUD insertion after medical abortion.}

Women's and staff perspectives on the best time to start with the IUD after medical abortion were informed by personal and clinical factors such as convenience, time saved, and contraceptive effectiveness.

\section{Preferences for immediate versus delayed insertion of the IUD}

Women generally described a preference for immediate insertion. Some from the delayed group felt the 3week delay would place them at risk of pregnancy, place additional demands on their time, and involve additional costs which meant they might not go for IUD insertion. This was echoed by providers. Preference for immediate insertion was expressed by two participants as follows.

"well I was so stressed when I found out that I couldn't put the IUD immediately because I thought what if in this process while I'm waiting for this IUD, what if something happened, coz it's very hard for us as married woman to use protection, specially to our husbands because they get to ask the questions...." (Immediate group, crossover to delayed, IUD inserted at 6 weeks by study clinician)

"I felt happy, I didn't have a problem that I was going to have it inserted because I wanted to have it inserted immediately, because I already heard how it works and just thought that if it works out for immediately, then I should have it inserted immediately and not waste time." (Immediate group, IUD expulsed and replaced at 6 weeks)

The increased efficiency of immediate insertion was expressed by a resident doctor as follows; 
"I think in terms of making sure it gets used, immediate insertion is easier for the woman and it cuts out one follow up visit which makes it easier to put in and use and I think to come back 6 weeks later for an insertion and then come back another 6 weeks later for a string check is very labour intensive. Whereas if it is put in at the time, it's in, she's more likely to use it and it just avoids second follow up". (Resident doctor, hospital)

\section{Barriers to immediate insertion of the IUD}

Although most healthcare providers considered immediate insertion a better option, they noted that staffing issues, specifically timely availability of providers competent to perform the IUD insertion and contraindications to immediate insertion could be barriers to an effective service.

One senior nursing sister expressed this as follows:

"only 1 registrar who is working the weekend, she has an emergency, she has theatre that she must take up, she has 2 wards that she must look after. And then apparently there's this patient that is waiting to go home, panicking because (the IUD insertion) it's not done, .......so if they delaying and all of that and she's done, she would rather take an injection or something else because the patient wants to go home". (Sister in charge of ward, hospital)

\section{Mechanisms to enable immediate insertion of the IUD}

To resolve staff shortages and lack of training, some healthcare staff suggested having a dedicated trained midwife to provide abortion and post-abortion care or alternatively training interns to do the IUD insertions as this should be within their competency and they would be more available.

"I think it would be solved by having a, a qualified nurse or qualified midwife, just one person who's responsible for this stream of care and then that would also mean that these patients can go home on the same day" (Study clinician)

"I think it would have been helpful if the interns was maybe trained to have done it because there's more interns than registrars". (Ward operations manager, hospital)

Theme 2: Follow up: Continuity of care and women's decisions at follow-up. Continuity of care following abortion

In the study's immediate arm protocol the abortion and postabortion care are rendered at the same place and by a provider familiar with the woman's reproductive history which meant she need not explain herself to a new healthcare provider:

"I mean, a great benefit is that women actually get the IUD inserted. And I think there's a second advantage because once women have it and are sort of in the system, they are also more likely to come back to their follow up, at least if you provide continuity of care. If you can offer that, women stay in the fold, you have a relationship and you say come back see me, I know your story, I know you've had an 
abortion, there's no problem with that, then I don't think they're as reluctant to come back for a check-up". (Study clinician)

\section{Women's decisions regarding the IUD during study follow- up}

In the RCT, $82 \%$ in the immediate and $21 \%$ in the delayed arm had the IUD inserted as planned. Despite a moderately high rate of partial expulsion which required removal of the original IUD in the immediate group, most of these women requested a replacement IUD. Some women in the delayed group asked for an IUD at the 6-week follow up even if they did not go to the CHF for insertion as planned. Thus, at 6 weeks $76 \%$ and $40 \%$ of women in the immediate versus delayed arm respectively, were using the original, a replacement or a newly placed IUD. Among the women in the delayed arm who did not have the IUD inserted as planned, many reverted to short term methods they had used before.

One woman who requested an IUD replacement at 6 weeks because the original IUD was malpositioned said the following:

"of course, I did have some problems after I put the IUD...... she said to me, it's low. Do you want us to take it right now, we put it right now or you want us to make another appointment). Then I said no, if you've got a chance you can do it now because I was already on a bed. And then it was not sore anywhere honestly, and then I decided immediately, take it out and put the new one.(Immediate group, IUD malpositioned, replaced at 6 weeks)

Another woman was planned for insertion at 3 weeks, but the provider was not available - she requested insertion at her 6-week follow-up; she described what happened as follows:

"I went to the clinic but the lady that was implanting the IUD wasn't there, so that's why I didn't put it in, they gave me the injection for 3 months and they said I must come back January... But I call the lady from [hospital name] and said, and she said it's fine, I can come, they will do it for me there...... (Delayed group; IUD inserted at 6 weeks by study clinician)

A woman in the delayed group changed her mind about the IUD but completed her follow-up interviews by phone. She explained her decision for the injectable method as being concerned about the possibility of expelling the device, not noticing it and becoming pregnant again.

"Yes, that while I have it in, it might fall out and what if I'm not as lucky as the others where when it falls out, they see it in their panties, and I don't see it and then I continue to have sex and then fall pregnant again, thinking that it's still in there whereas it had fallen out a long time ago." (Delayed group; injectable on discharge, no IUD at 6 weeks, 3 months or 6 months)

Many women who had the IUD inserted subsequently expressed happiness with the method and feelings of autonomy and liberation. 
'this IUD, it's quite good to me. I do not have even any cramps; my menstruation periods are normal. I do not have even any problem with it, it is fine, to me it's fine. I just like it, I'm not using anything, I can't even feel it, I can't even feel anything except when I put a finger inside for me to, to check. Yes, that's when I feel it you know inside. But it's as normal fine" (Immediate, got IUD as planned, IUD in situ throughout)

"ooh I felt really, I'm, I'm so happy, I feel free and stress free. I didn't, I don't have anything to worry about. About like kids, uh huh, the ones I have now it's fine, so I'm free, safe and free (Delayed group, IUD placed at 6 weeks by study clinician)

\section{Theme 3: Impact of supplementary activities on trial processes}

\section{Effect of counselling on IUD uptake and experience}

Women reported that the counselling supported contraception decision-making by providing information, encouragement, and reassurance which had a positive effect on IUD uptake and continuation. For instance, a participant noted:

"I took her word for it and I tried it and it's still working for me. She convinced me that it is a better option because she understands how I feel when I told her about the other ones I was on" (Immediate group, IUD expulsed and replaced at 6 weeks)

\section{Training of providers}

The need for competence and confidence in IUD insertion was a recurring theme expressed by providers, and emerged directly from the supplementary trial activities, notably the IUD insertion training sessions. These had a positive impact on motivation to provide immediate IUD insertion as expressed by one provider:

"well, I think if we had a session like that, we'd definitely be more confident in putting in the IUD and ja, I think it would improve the number of patients getting IUDs on time. I did do the training session that we had, and I'd been putting them in before that, but it was a nice refresher, I felt more confident after that" (Resident doctor, hospital)

\section{Effect of WhatsApp alerts to insert the IUD}

Providers considered the WhatsApp group a good mechanism to remind each other about patients needing the IUD inserted, especially on weekends, but also feared it may not be sustainable.

"I mean she's getting a TOP and she needs an IUD. So, she [the study clinician] would usually remind you the day before and especially on the weekends, I think it was really, really helpful because you wouldn't 
know that whether there's a TOP in the, in the ward or not coz they're totally closed off from everyone else" (Resident doctor, hospital)

\section{Communication between IUD study staff and women}

Communications by voice and text message from the study staff about follow-up were perceived positively by study participants. Women said this communication helped them buffer emotional difficulties and that it made them feel more informed and comfortable about using the IUD. They also noted that communication with the study staff seemed genuine and was non-condemnatory. One participant expressed this as follows:

"when they phone me, they phone me and then they ask me nicely, they not rude by the phone, they not forcing, like you must be there because you have that - it's a must, you must be there. They first arrange, how can we get you, will you be available any day, give us a date that you're going to be available, we can be available for you. That's another thing that makes me happy" (Immediate group, IUD in situ at 6 weeks)

\section{Impact of trial processes on participant or provider behaviour}

Providers generally thought that the trial processes were unlikely to have significantly influenced the RCT main outcomes, namely use and correct placement of the IUD 6 weeks after TOP. However, they noted the increased follow up at 6weeks among both study groups compared to usual and linked this to the compensation participants received for completing study visits and interviews

"it could have been compensation... I might not be $100 \%$ sure but definitely I think that it's mostly for the compensation. You must understand that most of, of our clients, I mean R100 means a lot to them". (Operations manager, abortion service, $\mathrm{CHC}$ )

\section{Theme 4: Contextual impacts on trial findings}

\section{Resource constraints and staff shortages}

Lack of availability of resources was a frustration expressed by healthcare staff and a potential barrier to providing the IUD immediately after the abortion, as expressed by two different doctors:

"it's uncomplicated, it's fairly easy. The only difficulty is just getting the necessary equipment.... And then at the beginning of the study, we also had a struggle with the instruments until they make a plan of getting their own instruments". (Resident doctor, hospital)

"I think there might be opposition from people because they see it as an extra job or chore to do, just because it takes more time than writing up a script for Petogen or the pill. But I don't think that should be a reason why it's not implemented" (Resident doctor, hospital)

\section{Women's perceptions of primary healthcare services}


Women explained reluctance to attend $\mathrm{CHCs}$ for follow-up because they feared facing a judgmental attitude on the part of healthcare providers. They also noted that waiting times at clinics were long and some described being turned away more than once.

"Like for example, going to the local clinics, sisters will ask you, ask you why, what happened, why you making abortion, you know this thing - like they will make you feel bad about doing it and, which is unprofessional, they not supposed to do it."

\section{Discussion}

We found that immediate insertion of the IUD is generally preferred by both women and clinical staff for reasons of convenience and continuity of care, and results in higher rates of clinical follow-up. Most women who receive the IUD are highly satisfied. The need for continuous training, communication mechanisms, instruments, and available staff in a highly pressured health system, as well as the rote administration of injectable as contraception, may be barriers to implementation.

\section{Strengths And Limitations}

The process evaluation was designed and performed concurrently with the clinical trial, the interviews as well as the main data analysis were performed by researchers not involved in trial procedures, and participants represented a broad range of roles and experiences. Bias in interpretation was mitigated by researcher triangulation but cannot be excluded. The identified barriers and facilitators to immediate IUD insertion are contextual to South Africa but many are likely applicable to similar settings.

Access to care is a multifaceted process which requires an interplay between laws and policies, provider attitudes, service delivery, and patient behaviours, in the absence of which access is jeopardized [6]. Implementing immediate insertion therefore requires an understanding of service delivery barriers as well as factors affecting women's health seeking behaviour and its relationship to service delivery.

\section{Women's health seeking behaviour}

In this study we found that if women receive an IUD after the abortion they are motivated to continue even if they require a replacement, and express high satisfaction with this method. Women expressed a clear preference for immediate over delayed IUD insertion and both women and staff predicted that delayed insertion was unlikely to occur because of the inconvenience, cost and potential negative experience of following up at referral clinics.

Women were reluctant to go to their referral clinics because they feared judgement on the part of providers and were therefore unwilling to tell their abortion history again. In contrast, the study communication and follow-up structure were perceived as convenient, and respectful, and being with 
providers with whom they were familiar was an incentive to follow-up. The additional study interventions were assessed as unlikely to have affected the main outcome of the study but may have influenced rates of follow-up. With the higher risk of IUD expulsion after immediate insertion it is important that these women attend their clinical follow-up after 4-6 weeks. The additional components of care offered during the trial such as personalized and convenient follow-up and non-judgmental communication, are therefore important to consider when planning the introduction of immediate insertion into routine service delivery. A review of current evidence supports that person-centred care is central to, and often lacking, in abortion care which impacts quality of care [7]. Contrary to expectations, cultural beliefs are not significant barriers to provision of non-judgmental service; active knowledge-seeking behaviour and the cumulative experience of working in the field of sexual and reproductive health are better predictors of quality care [8].

\section{Health service delivery of the IUD after medical abortion}

The group contraceptive counselling increased interest and uptake of the IUD. Use of the IUD is below $3 \%$ in South Africa. Staff are often unfamiliar with this method and women do not have family members or friends who can vouch for it.

A previous study has shown that the more knowledgeable a health care provider is about the IUD the more likely they are to recommend it [9]. In South Africa, injectable progestins, depotmedroxyprogesterone acetate (DMPA) and norethisterone enanthate (NET-EN), are used by $51 \%$ of women using contraception [2]. In this study we found that administration of an injectable postabortion was such a routine part of postabortion care that it was recurrently administered instead of contraceptive pills, in deviation of protocol, despite multiple reminders.

The study-initiated communication system with doctors was an important factor to ensure the IUD was inserted on time. Nurses highlighted the fact that doctors were absent from the ward during the time when most women would optimally receive their IUD for them to be able to go home the same day. Nurses and doctors also noted that staff shortages and lack of training were barriers to immediate IUD insertion. Making immediate IUD insertion part of routine postabortion care in the second trimester may require a restructuring of services to task-shift IUD insertion to either trained nurses or interns with higher presence in the ward, as well as regular training of clinic staff to maintain confidence in recommending and providing the IUD. Lack of recurring training is a known barrier to sustainable reproductive health services including IUD insertion $[10,11]$. Trained nurses and midwives can however provide equally safe, efficient and acceptable abortion and contraception care to physicians and their role may be better suited to person-centred services $[8,12,13]$.

\section{Conclusions}

Both women and providers support immediate insertion of the IUD after second trimester abortion. Adequate staffing and/or task-shifting of IUD insertion, available instruments and continuous training are important pre-requisites for implementation in the opinion of providers. For women, continuity of care, 
non-judgemental communication and convenient follow-up are important factors in ensuring uptake and adherence to follow-up.

\section{Declarations}

\section{Ethics approval}

Ethical approval for the RCT and for the PE were given by the University of Cape Town Human Ethics Research Council (RCT 007/2018; PE 821/2018) and the Western Cape Provincial Government (WC_201804_006)

\section{Consent for publication}

Not applicable for this paper

\section{Availability of data and materials}

All data will be made available by the corresponding author upon reasonable request

\section{Competing interests}

The authors declare no competing interests.

\section{Funding}

Anonymous donor (5290); National Research Foundation of South Africa / Swedish Foundation for International Cooperation in Research and Higher Education (170825260615/112631), Swedish Society of Medicine (SLS-783181), Swedish Society of Medical Research (P17-0214) University of Cape Town Research Committee.

\section{Authors' contributions}

DC and ME conceptualised, designed, and conducted the study. ODS performed the data analysis. DC, ME and ODS drafted and finalized the manuscript.

\section{Acknowledgements}

The authors would like to thank all study participants for their time and involvement in the research. Our thanks to Sofia Lopes for assistance with developing the protocol and interview guide and our interviewers - Dr Goretto and Ms. Nontuthuzelo Mvana and Ms. Kulthum Fataar.

\section{References}

1. The Guttmacher-Lancet Commission. Accelerate progress-sexual and reproductive health and rights for all: report of the Guttmacher-Lancet Commission. Executive summary. The Lancet. 2018;391. 
DOI 10.1016/S0140-6736(18)30293-9.

2. Chersich M, Wabiri N, Risher K, Shisana O, Celentano D, Rehle T, et al. Contraception coverage and methods used among women in South Africa: A national household survey. South African Medical Journal. 2017;107(4):307-14.

3. Moniz M, Chang T, Heisler M, Dalton VK. Immediate postpartum long-acting reversible contraception: the time is now. Contraception. 2017;95(4):335-8.

4. Hlongwa M, Mashamba-Thompson T, Makhunga S, Hlongwana K. Evidence on factors influencing contraceptive use and sexual behavior among women in South Africa: A scoping review. Medicine. 2020;99(12):e19490.

5. Moore GF, Audrey S, Barker M, Bond L, Bonell C, Hardeman W, et al. Process evaluation of complex interventions: Medical Research Council guidance. BMJ. 2015;350:h1258.

6. Benson J, Andersen K, Samandari G. Reductions in abortion-related mortality following policy reform: evidence from Romania, South Africa and Bangladesh. Reproductive Health. 2011;8(1):39.

7. Altshuler AL, Whaley NS. The patient perspective: perceptions of the quality of the abortion experience. Curr Opin Obstet Gynecol. 2018;30(6):407-13. doi:10.1097/GC0.0000000000000492.

8. Tumwine G, Agardh A, Gummesson C, Okong P, Östergren PO. Predictors of health care practitioners' normative attitudes and practices towards sexual and reproductive health and rights: a crosssectional study of participants from low-income countries enrolled in a capacity-building program. Glob Health Action. 2020;13(1):1829827. doi:10.1080/16549716.2020.1829827.

9. Kohn JE, Hacker JG, Rousselle MA, Gold M. Knowledge and likelihood to recommend intrauterine devices for adolescents among school-based health center providers. J Adolesc Health. 2012;51(4):319-24.

10. O'Shea LE, Hawkins JE, Lord J, Schmidt-Hansen M, Hasler E, Cameron S, et al. Access to and sustainability of abortion services: a systematic review and meta-analysis for the National Institute of Health and Care Excellence-new clinical guidelines for England. Hum Reprod Update. 2020;26(6):886-903. doi:10.1093/humupd/dmaa026.

11. Thompson EL, Vamos CA, Logan RG, Bronson EA, Detman LA, Piepenbrink R, et al. Patients and providers' knowledge, attitudes, and beliefs regarding immediate postpartum long-acting reversible contraception: a systematic review. Women Health. 2020;60(2):179-96. doi: 10.1080/03630242.2019.1616042. Epub 2019 May 23.

12. Mainey L, O'Mullan C, Reid-Searl K, Taylor A, Baird K. The role of nurses and midwives in the provision of abortion care: A scoping review. J Clin Nurs. 2020;29(9-10):1513-26. doi:10.111/jocn.15218. Epub 2020 Feb 29.

13. Polus S, Lewin S, Glenton C, Lerberg PM, Rehfuess E, Gülmezoglu AM. Optimizing the delivery of contraceptives in low- and middle-income countries through task shifting: a systematic review of effectiveness and safety. Reprod Health. 2015;12:27. 10.1186/s12978-015-0002-2. doi).

\section{Supplementary Files}


This is a list of supplementary files associated with this preprint. Click to download.

- COREQDC.docx 